\title{
NEW ANALYSIS OF WOLF-RAYET INFRARED LINES
}

\author{
P.R.J. EENENS \\ Instituto de Astronomía, UNAM, Apartado Postal 877, Ensenada, B.C., Mexico \\ and \\ P.M. WILLIAMS \\ Royal Observatories, Edinburgh EH9 $3 H J$, United Kingdom
}

\begin{abstract}
New infrared spectra of Wolf-Rayet stars are analysed to derive $\mathrm{C} / \mathrm{He}$ abundance ratios and ionization ratios. These ratios are discussed in relation with subtype, terminal velocity and local metallicity. They are compared with the predictions of evolutionary models.
\end{abstract}

Key words: stars: Wolf-Rayet - evolution - atmospheres

This study is part of an ongoing programme of observation and analysis of infrared spectra of galactic WR stars. Here we present new infrared (1.0 1.2 and $2.1-2.5 \mu \mathrm{m}$ ) spectra of Wolf-Rayet stars obtained with CGS4 at UKIRT. Together with previous UKIRT spectra (Eenens, Williams \& Wade 1991), these provide a set of data for 14 stars of all WC subtypes and one WO subtype. More observations will be added in the near future.

The recombination analysis used to derive relative abundances is described by Hummer, Barlow \& Storey (1982) and Eenens \& Williams (1992). The helium lines near 1.042, 1.094, 2.165, 2.189 and $2.347 \mu \mathrm{m}$ and the carbon lines near $1.025,1.055,1.191,2.32,2.427$ and $2.48 \mu \mathrm{m}$ are used to derive $\mathrm{C}^{4+} / \mathrm{C}^{3+}$ and $\mathrm{C} / \mathrm{He}$ ratios (by number). The strong He II feature at 1.0125 $\mu \mathrm{m}$ does not give consistent abundances and was not used.

The ionization of the wind, as measured by the $\mathrm{C}^{4+} / \mathrm{C}^{3+}$ ratio, decreases with increasing subtype. This trend corresponds to the intention behind the subtypes definitions, although line optical depth and wind composition are also affecting the ratios of the classifying optical lines. The two stars characterized by non-thermal radio emission (WR140 and WR146) are outof-line, their ionization being too high for their subtype, probably due to wind-wind collisions in WR+O binary systems (Williams et al. 1992). There is a loose inverse correlation between ionization ratio and terminal velocity, as expected in a radiatively driven wind. These two correlations linking the ionization ratio with the subtype and with the wind velocity respectively should be viewed together with the correlation between terminal velocity and subtype observed by Eenens \& Williams (1994).

The $\mathrm{C} / \mathrm{He}$ abundance ratio decreases with increasing WC subtype and is the highest for the WO star. Once again WR146 stands out, as its subtype 
classification is affected by its anomalously strong wind. The present data do not show the increase of $\mathrm{C} / \mathrm{He}$ with larger galactocentric distance (lower metallicity) predicted by evolutionary models (Maeder 1992).

When comparing our $\mathrm{C} / \mathrm{He}$ ratios with those predicted in the $Z=0.02$ models of Schaller et al. (1992), a good agreement is found between our results for the WC4-5 subtypes and their predictions for stages 34-38 of the $60-\mathrm{M}_{\odot}$ model, while the WO value would correspond to stage 41 . However we find that in all their models (for different initial masses), the stage corresponding to later WC subtypes is only represented as a very rapid transition, in disagreement with the observed number ratios of WC subtypes. The more recent models of Meynet et al. (1994), which are characterized by higher mass-loss rates during the WNL phase, provide a better overall agreement, especially the $60-\mathrm{M}_{\odot}$ model. Stages $28-33$ correspond to WC6-9 subtypes, stages $36-39$ to the WC4-5 subtypes and stage 51 to the WO2 subtype. Their $40-\mathrm{M}_{\odot}$ model does not reach the high value we derive for the WO star. Therefore our analysis is in favour of the increased mass-loss rates included in the latest models, supports an initial mass of $60 \mathrm{M}_{\odot}$ and indicates that early WC subtypes are more evolved than late ones, and that the WO subtype is the most evolved.

\section{References}

Eenens, P.R.J., Williams, P.M., Wade, R. 1991, MNRAS 252, 300

Eenens, P.R.J., Williams, P.M. 1992, MNRAS 255, 227

Eenens, P.R.J., Williams, P.M. 1994, MNRAS in press

Hummer, D.G., Barlow, M.J., Storey, P.J. 1982, in: C. de Loore \& A.J. Willis (eds.), WolfRayet Stars: Observations, Physics, Evolution, Proc. IAU Symp. No. 99 (Dordrecht: Reidel), p. 79

van der Hucht, K.A., Hidayat, B., Admiranto, A.G., Supelli, K.R., Doom, C. 1988, $A \& A$ 199,217

Meynet, G., Maeder, A., Schaller, G., Schaerer, D., Charbonnel, C. 1994, A\&A Suppl. 103,97

Maeder, A. 1992, $A \& A$ 264, 105

Schaller, G., Schaerer, D., Meynet, G., Maeder, A. 1992, A\&A Suppl. 96, 269

Williams, P.M., van der Hucht, K.A., Bouchet, P., Spoelstra, T.A.Th., Eenens, P.R.J., Geballe, T.R., Kidger, M.R., Churchwell, E.B. 1992, MNRAS 258, 461 